\title{
Analysis of IMP3 expression in primary tumor and stromal cells in patients with colorectal cancer
}

\author{
XIAOPING HUANG ${ }^{1 *}$, QINGZHU WEI ${ }^{2 *}$, JIANGHUAN LIU $^{2}$, HONGLING NIU $^{1}$, GANG XIAO $^{1}$ and LIXIN LIU ${ }^{1}$ \\ Departments of ${ }^{1}$ General Surgery and ${ }^{2}$ Pathology, The Third Affiliated Hospital of \\ Southern Medical University, Guangzhou, Guangdong 510630, P.R. China
}

Received April 18, 2016; Accepted July 7, 2017

DOI: $10.3892 /$ ol.2017.7161

\begin{abstract}
Insulin-like growth factor II mRNA-binding protein 3 (IMP3) is an oncofetal protein upregulated in tumor cells during carcinogenesis. The aim of the present study was to investigate the expression status of IMP3 in colorectal cancer (CRC) tissues and its clinical significance. Immunostaining was performed in 130 CRC samples, the association of IMP3 expression with clinicopathological characteristics was assessed and 58 patients were selected for survival analysis. To the best of our knowledge, the present study describes for the first time the expression of IMP3 in tumor stromal components of CRC. Stromal expression of IMP3 was detected in 24/130 (18.5\%) CRC tissue specimens and was associated with tumor-node-metastasis (TNM) stage (stage III-IV, $\mathrm{P}=0.003)$, lymph node metastasis $(\mathrm{P}=0.006)$, lympho-vascular invasion $(\mathrm{P}=0.003)$, tumor border $(\mathrm{P}=0.013)$. Tumoral expression of IMP3 was detected in 94/130 (72.3\%) of CRC specimens and was associated with T classification (T3-T4, $\mathrm{P}=0.027$ ), tumor-node-metastasis (TNM) stage (stage III-IV, $\mathrm{P}=0.011)$, lymph node metastasis $(\mathrm{P}=0.048)$, tumor budding ( $>10$ buds, $\mathrm{P}=0.005)$. Further study indicated that patients with IMP3 expressed in tumor cells and tumor stroma tend to have poorer overall survival rates $(\mathrm{P}=0.02$ and $\mathrm{P}=0.06$, respectively). Moreover, tumoral expression of IMP3 and TNM stage were identified to be independent prognostic factors in CRC. IMP3 was not only expressed in tumor cells but also in stroma cells. Stromal expression of IMP3 was associated with lymph node metastasis and advanced tumor TNM stage. Moreover, the survival analysis indicated that there is a significant association between IMP3 expression in tumor cells and a poorer overall
\end{abstract}

Correspondence to: Dr Lixin Liu, Department of General Surgery, The Third Affiliated Hospital of Southern Medical University, 183 Zhongshan Dadaoxi, Guangzhou, Guangdong 510630, P.R. China E-mail: liuliixn@tom.com

*Contributed equally

Key words: colorectal cancer, insulin-like growth factor II mRNA-binding protein 3, stroma, lymph node metastasis, tumornode-metastasis stage, survival survival rate in patients with CRC. The expression of IMP3 maybe a predicted factor for CRC patient.

\section{Introduction}

Colorectal cancer (CRC) is the third most common type of diagnosed cancer and a major cause of mortality in western countries (1). Although resection of primary CRC can be curative when the disease is localized, distant metastasis remains the primary cause of treatment failure and mortality from cancer. The existing standardized pathological staging systems do not reflect the exact biological behavior of carcinoma, which may be associated with tumor metastasis and recurrence. In the past decades, treatment and molecular characteristics relating to prognosis of CRC have been focused mainly on malignant cancer cells. However, recent studies revealed that $\mathrm{CRC}$ progression was mediated in many aspects by tumor stroma (2), and improvements in survival have been observed in patients with metastatic CRC using the therapeutic strategy of targeting tumor stroma (3).

Insulin-like growth factor II mRNA-binding protein 3 (IMP3; IGF2BP3) is a member of the insulin-like growth factor II mRNA-binding proteins. IMP3 is known as an oncofetal protein, which has been only been detected in human cancer tissues and early human embryogenesis, but not in normal tissues of adults (4). Two known functions of IMP3 are regulation of mRNA stability and subcellular localization (5), and further studies have indicated that IMP3 promotes tumor cell proliferation, adhesion and invasion (6-8). Recent studies revealed that IMP3 expression in tumor cells was a marker of poor prognosis in CRC patients (9-12). However, limited information is known about whether stromal expression of IMP3 is of any clinical relevance in CRC.

Tumor stroma is a circuitous ecosystem consisting of an extracellular matrix (ECM) scaffold that is populated by cancer-associated fibroblasts (CAF), vascular space-related cells, and diverse innate and adaptive immune response cells (13). According to the 'seed and soil' hypothesis, tumor cells and stromal cells co-evolve (14). Metastasis is determined by a number of complex interactions between tumor cells (the 'seed') and their stroma (the 'soil') (15). Tumor stoma is pivotal not only to tumor initiation, malignant progression and metastasis, but also to response to therapy (16). Massoner et al (17) demonstrated that stroma 
was the main source of IMP3 in the prostate, suggesting that IMP3 acted as a mediator of stromal-epithelial interactions. Epithelial mesenchymal transition (EMT) is proposed as a critical mechanism for the acquisition of malignant phenotypes by epithelial cells (18). In colorectal cancer, tumor cells that have undergone EMT are characterized histologically by the presence of tumor buds, which are defined as single cells or small clusters of de-differentiated tumor cells at the invasive front (19). Tumor budding is predictive of lymph node metastasis, vascular and lymphatic invasion, distant metastasis, local recurrence and poor disease-specific survival time (20).

In the present study, expression of IMP3 in CRC was evaluated by immunohistochemistry, and it was observed that IMP3 expression was not only abundant in the cytoplasm of tumor cells, but expression was also detected in stroma cells. The pattern of IMP3 staining in CRC specimens was examined in order to investigate its role in the progression and survival of patients with CRC.

\section{Materials and methods}

Patientsandtumorsamples.Formalin-fixed,paraffin-embedded samples were obtained following surgical resection from 130 patients (70 males and 60 females; mean age, 61.1 years; range, 24-88 years) with primary colorectal carcinoma who did not undergo any radiotherapy and chemotherapy prior to surgery in the Department of Pathology at the Third Affiliated Hospital of Southern Medical University (Guangzhou, China) between January 2004 and December 2014. During follow-up, 58 patients had been followed for $>60$ months or until mortality at the end of follow-up, 27 patients succumbed to disease and 103 patients survived. Clinical data was obtained from follow-up records including age, gender, tumor location, tumor size and survival time. The experimental protocol was approved by the Human Ethics Review Committee of the Third Affiliated Hospital of Southern Medical University. Informed consent was obtained from all patients.

Histological examination and tumor stage. The tumor located on the right side [30 cases, cecum (12 cases), ascending colon (12 cases) and hepatic flexure (6 cases)] and left side [100 cases, splenic flexure (16 cases), descending colon (23 cases) and sigmoid colon (61 cases)], respectively. All specimens were routinely fixed in $10 \%$ buffered formalin and embedded in paraffin at $70^{\circ} \mathrm{C}$, put on ice for $10 \mathrm{~min}$, and $4 \mu \mathrm{m}$ thick sections were cut and stained with hematoxylin and eosin ( $\mathrm{H}$ and $\mathrm{E}$ ) for 2-3 $\mathrm{min}$ at room temperature. All $\mathrm{H}$ and $\mathrm{E}$ slides of specimens were re-evaluated, and the diagnosis was confirmed by two experienced pathologists. Histological subtype, presence of lympho-vascular invasion, lymph node metastasis, tumor border and tumor budding were assessed. The histological grade was assessed according to the World Health Organization 2010 criteria (21). The tumor stage was based on pathological findings according to the American Joint Committee on Cancer (AJCC) guidelines (22). Tumor stage was classified as stage I (T1-2 N0 M0, 16 cases), II (T3-4 N0 M0, 47 cases), III (T1-4 N1-2 M0, 38 cases) and IV (T1-4 N0-3 M1, 29 cases).

Tumor budding was defined as dedifferentiated single cells or clusters of $<5$ cells at the invasive tumor front. The extent of tumor budding was assessed on caudal type homeobox 2 (CDX2)-stained whole tissue sections as described previously by Karamitopoulou et al (23). The CDX2-stained whole tissue sections were first examined at low magnification (x4) and the area with the highest density of peritumoral budding was selected for counting. The average number of buds was counted in ten high power fields (magnification, $x 40$ ). Specimens were divided into two groups according to the average number of budding: Low grade ( $\leq 10$ buds) and high grade ( $>10$ buds).

Immunohistochemical analysis. Immunohistochemical staining was performed on $4 \mu \mathrm{m}$ thick sections. In brief, the sections were deparaffinized and rehydrated. Heat-induced antigen retrieval was performed at $95^{\circ} \mathrm{C}$ for $15 \mathrm{~min}$ in citrate buffer ( $\mathrm{pH}$ 6.0). Endogenous peroxidase activity was quenched with $3 \%$ hydrogen peroxide solution. The sections were immunostained using a monoclonal mouse anti-human antibody against IMP3 (cat. no. M362629-2; clone 69.1; dilution, 1:100; Dako; Agilent Technologies, Inc., Santa Clara, CA, USA) and CDX2 (cat. no. GT201907; clone EPR2764Y; dilution, 1:100; Gene Tech Company, Ltd., Shanghai, China). The GTVision ${ }^{\mathrm{TM}}$ Detection system (Gene Tech Company, Ltd.) and 3,3'-diamino-benzidine (Gene Tech Company, Ltd.) were used to detect antibody-conjugated peroxidase activity. The sections were then counterstained with hematoxylin for 2-3 min at room temperature, dehydrated and mounted.

Evaluation of immunohistochemical staining. Immunostaining was assessed by two experienced pathologists, who were blinded to the clinical data of the patients. Stromal cells positive for cytoplasmic IMP3 staining were considered IMP3-positive. IMP3 expression in tumor cells was considered positive if IMP3 staining in tumor cells was $>10 \%$. Nuclear staining for CDX2 was considered positive in resection specimens (i.e. a majority of cancer cells exhibited widespread nuclear expression of CDX2; those exhibiting faint or no nuclear expression were considered CDX2-negative).

Statistical analysis. Statistical analyses were performed using SPSS (version 13.0; SPSS, Inc., Chicago, IL, USA). The association between IMP3 expression in tumor stroma and tumor cells was analyzed using Pearson $\chi^{2}$ test. The association between IMP3 expression (in tumor stroma and tumor cells) and clinicopathological parameters were evaluated by Pearson $\chi^{2}$ and Fisher's exact tests. The Kaplan-Meier method and the log-rank test were used to analyze the associations between IMP3 expression (in tumor stroma and tumor cells) and the overall survival time of patients. A Cox regression model was used for univariate and multivariate analyses to determine the independent significance of relevant clinical covariates. Two tailed $\mathrm{P}<0.05$ was considered to indicate a statistically significant difference.

\section{Results}

Patient results. A total of 130 patients with CRC were available for review between 2004 and 2014 (mean age, 61.1 years; range, 24-88 years). The end date of the follow-up study for conducting the analysis was 1 January 2015 (mean duration of follow-up, 52.5 months; range, 1-132 months). A total of 
Table I. Association between stromal and tumoral expression of IMP3 and clinicopathological characteristics in 130 patients with colorectal cancer.

IMP3 expression in tumor stroma

Characteristic

Colorectal cancer

Age, years

$\leq 45$

$>45$

Gender

Female

Male

Tumor location

Left sided

Right sided

Tumor size, diameter $(\mathrm{cm})$

$\leq 4$

$>4$

Histological grade

Well and moderately differentiated

Poorly differentiated

Histological subtype

Non-mucinous

Mucinous

$\mathrm{T}$ classification

T1-T2

T3-T4

TNM stage

I-II

III-IV

Lymph node metastasis

Absent

Present

Tumor border

Infiltrating

Pushing

Tumor budding

Low ( $\leq 10$ buds)

High (>10 buds)

Lympho-vascular invasion

Absent

Present
Total Positive (\%) $\quad$ Negative (\%) P-value

$\begin{array}{rcc}130 & 24(18.5) & 106(81.5) \\ 23 & 2(8.7) & 21(91.3) \\ 107 & 22(20.6) & 85(79.4)\end{array}$

$$
60
$$

60
70

$60 \quad 8(13.3)$

$52(86.7)$

$16(22.9)$

$54(77.1)$

$100 \quad 21(19.6)$

$85(85.0)$

$30 \quad 3(13.0)$

$21(87.0)$

$59 \quad 15(15.0)$

85 (85.0)

$71 \quad 9(30.0)$

$21(70.1)$

$107 \quad 9(15.3)$

$50(84.7)$

$23 \quad 15(21.1)$

$56(78.9)$

$115 \quad 24(20.9)$

$15 \quad 0(0)$

$91(79.1)$

15 (100)

20

1 (50)

19 (95.0)

110

23 (20.9)

87 (79.1)

$63 \quad 5(7.9)$

$67 \quad 19(28.4)$

$58(92.1)$

48 (71.6)

$66 \quad 6(9.1)$

$60(90.9)$

64

$18(28.1)$

46 (71.9)

$90 \quad 22(24.4) \quad 68(75.6)$

$40 \quad 2(5.0) \quad 38(95.0)$

$71 \quad 11(15.5) \quad 60(84.5)$

$59 \quad 13(22.0) \quad 46(78.0)$

$57 \quad 4(7.0)$

$53(93.0)$

$73 \quad 20(27.4) \quad 53(72.6)$

0.244

0.181

0.104

0.497

0.566

0.130

0.121

0.003

0.006

0.013

0.371

0.003
IMP3 expression in tumor cells

Positive (\%) Negative (\%) P-value

$94(72.3) \quad 36(27.7)$

0.445

$15(65.2) \quad 27(34.8)$

$79(73.8) \quad 28(26.2)$

$41(68.3) \quad 19(31.7)$

$53(75.7) \quad 17(24.3)$

$75(75.0) \quad 25(25.0)$

$19(63.3) \quad 11(36.7)$

$39(66.1) \quad 20(33.9)$

$55(77.5) \quad 16(22.5)$

$74(69.2) \quad 33(30.8)$

$20(87.0) \quad 3(13.0)$

$82(71.3) \quad 33(28.7)$

$12(80.0) \quad 3(20.0)$

$10(53.4) \quad 10(46.6)$

$84(76.4) \quad 26(23.6)$

39 (61.9) $24(38.1)$

$55(82.1) \quad 12(17.9)$

$43(65.2) \quad 23(34.8)$

$52(81.2) \quad 12(18.8)$

$70(77.8) \quad 20(22.2)$

$24(60.0) \quad 16(40.0)$

0.005

$44(62.0) \quad 27(38)$

$50(84.7) \quad 9(15.3)$

$37(64.9) \quad 20(35.1)$

$57(78.1) \quad 16(21.9)$

0.432

0.247

0.027

0.011

0.048

0.055

0.116
IMP3, insulin-like growth factor II mRNA-binding protein 3; TNM, tumor-node-metastasis.
58 patients were followed for $>60$ months or until mortality. At the end of follow-up, 27 patients succumbed to disease and 103 patients survived. Clinicopathological characteristics of the patients and tumor characteristics are summarized in Table I.

Immunohistochemical staining in CRC tissue samples. Immunostaining of IMP3 was performed in CRC tissue samples (Fig. 1). IMP3 expression was not only detected in the tumor cells region, but also in the cytoplasm of tumor stroma cells (Fig. 1B). Notably, it was observed that stromal expression of IMP3 was primarily expressed in the spindle cells of the tumor stroma (Fig. 1C). Of the 24 specimens positive for IMP3 expression in the stroma, positive expression in the tumor cells was also detected in 18 cases. However, no statistically significant association was identified between IMP3 expression in the tumor cells and tumor stroma (0.807; Table II). Positive stromal 
Table II. Concordance of IMP3 expression between tumor cells and tumor stroma.

\begin{tabular}{lcrr}
\hline & \multicolumn{2}{c}{ Tumoral IMP3 expression, $\mathrm{n}$} & \\
\cline { 2 - 4 } Stromal IMP3 expression & Positive & Negative & Total, $\mathrm{n}$ \\
\hline Positive & 18 & 6 & 24 \\
Negative & 76 & 30 & 106 \\
Total & 94 & 36 & 130 \\
\hline
\end{tabular}

IMP3, insulin-like growth factor II mRNA-binding protein 3.

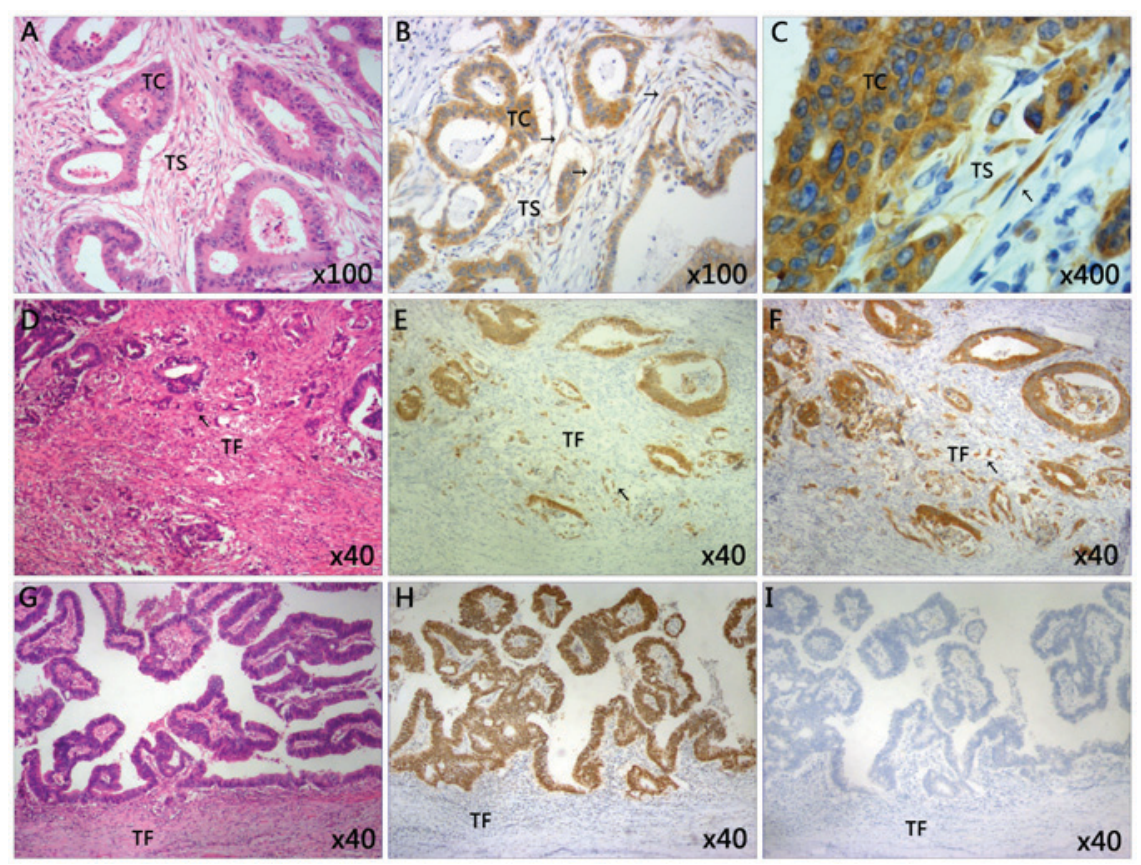

Figure 1. Representative images of immunohistochemical staining of tissues from patients with CRC. (A) The classic microscopic features of colorectal cancer with hematoxylin and eosin staining. The chromatin in the nucleus was stained violet blue and the cytoplasm and extracellular matrix were stained red. Magnification, x100. (B) High expression of IMP3 in tumor cells and tumor stroma cells. Magnification, x100. (C) The IMP3-positive cells in tumor stroma closely resemble spindle cells. Magnification, x400. (D) High grade of tumor budding stained with hematoxylin and eosin in the TF. Magnification, x40. (E) The present study observed the tumor buds using CDX2 staining. CDX2 stained the nucleus brown. The mean number of buds was counted using ten high power fields. Specimens were divided into two groups according to the average number of buds: Low grade ( $\leq 10$ buds) and high grade (>10 buds). Here the image revealed the high grade tumor budding with CDX2 staining in the TF. Magnification, x40. (F) High expression of tumor budding stained with IMP3 in tumor front of CRC. Magnification, x40. (G) Low grade tumor budding with hematoxylin and eosin staining in the TF. Magnification, x40. (H) Low grade of tumor budding stained with CDX2 in tumor front of CRC. Magnification, x40. (I) Negative expression of IMP3 in tumor tissue and tumor front. Magnification, $\mathrm{x} 40$. Slides A, D and G were stained with hematoxylin and eosin. CDX2, caudal type homeobox 2; CRC, colorectal cancer; IMP3, insulin-like growth factor II mRNA-binding protein 3; TC, tumor cells; TS, tumor stroma; TF, tumor front.

expression of IMP3 (stroma cells positive for cytoplasmic IMP3 staining) was detected in 24/130 patients (18.5\%), and negative expression was observed in $106 / 130$ patients $(81.5 \%)$. By contrast, positive tumoral expression of IMP3 (IMP3 staining in $>10 \%$ of tumor cells) was detected in 94/130 cases of CRC (72.3\%), and negative expression was detected in 36/130 patients (27.7\%) (Table I). High tumor budding (Fig. 1E) was observed in 59/130 (45.4\%) patients, whereas low tumor budding (Fig. 1H) was detected in 71/130 (54.6\%) cases (Table I). High tumor budding with positive IMP3 expression was also observed in tumor front of CRC (Fig. 1F).

Association between stromal expression of IMP3 and clinicopathological characteristics. Stromal expression of IMP3 was associated with TNM stage (stage III-IV, $\mathrm{P}=0.003$ ), lymph node metastasis $(\mathrm{P}=0.006)$, lympho-vascular invasion $(\mathrm{P}=0.003)$ and tumor border (infiltrating vs. pushing, $\mathrm{P}=0.013$ ). No statistically significant associations were observed between IMP3 expression in tumor stroma and age $(\mathrm{P}=0.244)$, gender $(\mathrm{P}=0.181)$, tumor location $(\mathrm{P}=0.104)$, tumor size $(\mathrm{P}=0.497)$, histological grade $(\mathrm{P}=0.566)$, histological subtype $(\mathrm{P}=0.130)$, T-classification (T1-2 vs. T3-4, $\mathrm{P}=0.121)$ and tumor budding $(\mathrm{P}=0.371)$ (Table I).

Associations between tumoral expression of IMP3 and clinicopathological characteristics. Tumoral expression of IMP3 was significantly associated with T-classification (T1-2 vs. T3-4, P=0.027; Table I), TNM stage (stage I-II vs. III-IV, 
Table III. Univariate and multivariate analyses of the associations between prognostic variables in 130 patents with colorectal carcinoma.

\begin{tabular}{|c|c|c|c|c|c|c|}
\hline \multirow[b]{2}{*}{ Parameters } & \multicolumn{2}{|c|}{ Univariate analysis } & \multirow[b]{2}{*}{ P-value } & \multicolumn{2}{|c|}{ Multivariate analysis } & \multirow[b]{2}{*}{ P-value } \\
\hline & HR & $95 \% \mathrm{CI}$ & & HR & $95 \% \mathrm{CI}$ & \\
\hline Tumoral expression of IMP3 & & & & & & 0.029 \\
\hline Negative vs. positive & 11.891 & $1.606-88.074$ & 0.015 & 9.356 & $1.250-70.048$ & \\
\hline TNM stage & & & & & & 0.045 \\
\hline I- II vs. III -IV & 7.195 & 2.471-20.949 & $<0.001$ & 6.178 & $1.037-36.800$ & \\
\hline Lymph node metastasis & & & & & & 0.567 \\
\hline No vs. yes & 4.068 & $1.708-9.689$ & 0.002 & 0.511 & $0.051-5.0913$ & \\
\hline Lympho-vascular invasion & & & & & & 0.402 \\
\hline No vs. yes & 3.973 & $1.499-10.530$ & 0.006 & 0.402 & $0.358-12.913$ & \\
\hline
\end{tabular}

Analyses were performed using the Cox regression model. CI, confidence interval; HR, hazard ratio; IMP3, insulin-like growth factor II mRNA-binding protein 3 .
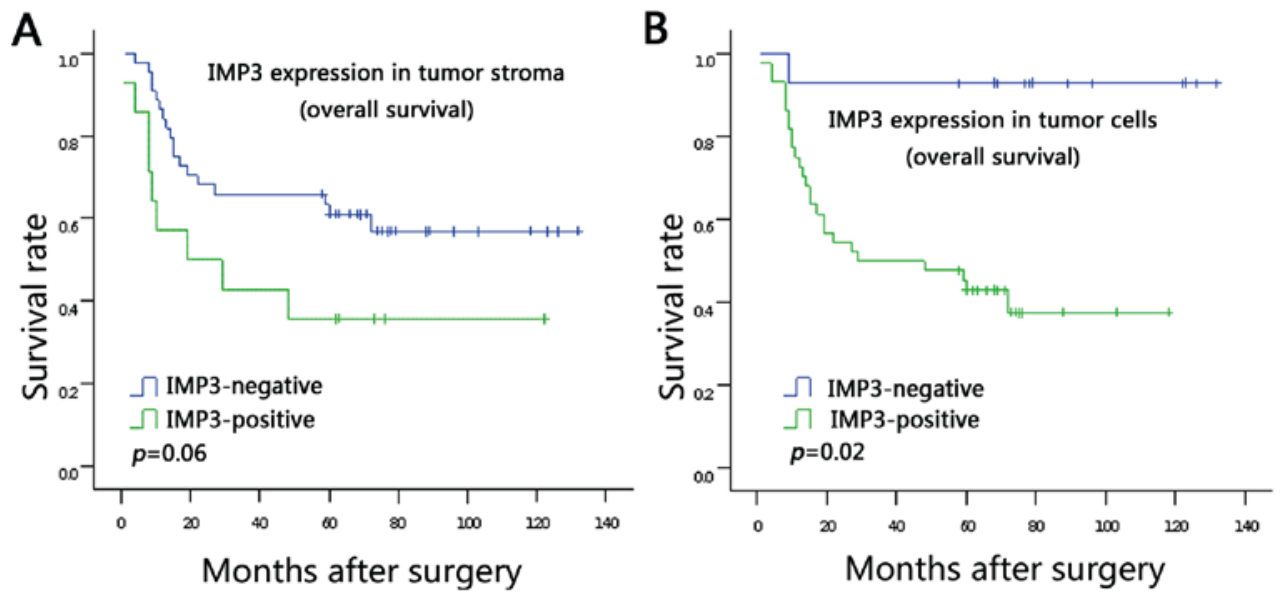

Figure 2. Kaplan-Meier analysis of overall survival rate in 130 patients with CRC in relation to IMP3 expression. (A) Patients with positive IMP3 expression in tumor stroma tend to have a lower 5-year survival rate compared with patients with a lower 5-year survival rate. $\mathrm{P}=0.06$. (B) There was an association between overexpression of IMP3 in tumor cells and poorer 5-year survival rate. P=0.02. CRC, colorectal cancer; IMP3, insulin-like growth factor II mRNA-binding protein 3.

$\mathrm{P}=0.011$ ), lymph node metastasis (absent vs. present, $\mathrm{P}=0.048$ ) and tumor budding (low vs. high, $\mathrm{P}=0.005$ ). Additionally, IMP3 expression was significantly associated with CDX2 expression in tumor cells and tumor budding (all $\mathrm{P}=0.005$ ), which means tumor budding with CDX2 staining was also positive for IMP3, (Fig. 1E, F, H and I). Furthermore, there was no significant association between IMP3 expression and age $(\mathrm{P}=0.445)$, gender $(\mathrm{P}=0.432)$, tumor location $(\mathrm{P}=0.247)$, tumor size $(\mathrm{P}=0.171)$, histological grade $(\mathrm{P}=0.122)$, histological subtype $(\mathrm{P}=0.759)$, lympho-vascular invasion $(\mathrm{P}=0.116)$ and tumor border $(\mathrm{P}=0.055)$ (Table I).

Survival analysis. The associations between IMP3 expression in the tumor stroma and tumor cells and survival rate were analyzed using Kaplan-Meier analysis (Fig. 2A and B). It was demonstrated that patients with positive IMP3 expression in tumor cells had a poorer survival rate compared with patients with negative IMP3 expression in tumor cells (log rank
$\mathrm{P}=0.02$; Fig. 2B). The data did not show statistically significant association between stromal expression of IMP3 and survival rate, but a trend is evident from the survival curve: That patients with stromal expression of IMP3 tend to have poor survival rates ( $\log$ rank $\mathrm{P}=0.06$; Fig. $2 \mathrm{~A}$ ). Moreover, in a univariate analysis based on the Cox regression model, tumoral expression of IMP3 $(\mathrm{P}=0.015)$, TNM stage $(\mathrm{P}<0.001)$, lymph node metastasis $(\mathrm{P}=0.002)$ and lympho-vascular invasion $(\mathrm{P}=0.006)$ were observed to be associated with poor prognosis (Table III). However, multivariate survival analysis using the Cox regression model indicated that tumoral expression of IMP3 $(\mathrm{P}=0.029)$ and TNM stage $(\mathrm{P}=0.045)$ were independent prognostic factors (Table III).

\section{Discussion}

Distant metastasis and recurrence are still the main causes of mortality in patients with CRC (1). Investigation of metastatic 
mechanisms and identification of molecular targets remains a key issue faced in CRC research. Tumor stroma has a crucial role in tumor progression, which provides an interface between malignant cells and host tissue (24). The balance of host-tumor interdependency is able to modulate the phenotype of a tumor to influence the outcome of the disease (25). IMP3 expression has been verified to be present predominantly in the tumor cells of multiple malignancies including CRC, and its presence has been associated with poor survival following surgical resection (9-12). However, little is known about the role of stromal IMP3 in CRC progression.

To the best our knowledge, the present study elucidated for the first time that IMP3 expressed in tumor stroma cells in CRC was associated with TNM stage, lymph node metastasis, lympho-vascular invasion and tumor border. These findings indicated that stromal expression of IMP3 may be used as a potential marker for lymph node metastasis and TNM stage. The stromal expression of IMP3 may be useful in identifying CRC patients with a poor prognosis though the present data did not indicate a statistical significant association between stromal expression of IMP3 and a poor survival $(\mathrm{P}=0.06)$, which may be due to a small sample size (follow-up > 60 month, 58 cases) used in the study. Therefore, large scale studies are warranted. IMP3 expression has been detected in the stroma, particularly reactivated stroma which was the main source of IMP3 in the prostate, suggesting that this peptide acts as a mediator of stromal-epithelial interaction (17). Further study showed that IMP3 was essential for transforming growth factor $\beta 1$-mediated differentiation. The dysregulation of the stromal IGF axis, in particular elevated IMP3 expression, has a crucial role in fibroblast-to-myofibroblast differentiation in the diseased prostatic stroma (26). Since the abundance of myofibroblasts in cancer-associated stroma may be an useful indicator of disease recurrence in CRC patients (27), the findings in the present study lead to the hypothesis that IMP3 may contribute to the recruitment of CAFs to promote tumor invasion and metastasis. The molecular mechanism of stromal IMP3 in the CRC progression would be the focus of further study.

Consistent with previous findings (9-12), the present study demonstrated that positive IMP3 expression in tumor cells predicted a poor survival in CRCs $(\mathrm{P}=0.02)$. Moreover, to the best of our knowledge, the present study was the first to associate IMP3 expression in tumor cells with tumor budding $(\mathrm{P}=0.005)$. Tumor budding is not a static, histological feature; it represents a snapshot of a dynamic process undertaken by an aggressive tumor with the potential to disseminate and metastasize (28). Tumor budding was recommended as an additional prognostic factor according to the AJCC (29). Furthermore, it was detected in the present study that tumor budding in the tumor front was also positive for IMP3 staining. Taken together, the strong association indicated that IMP3 may have a vital role in EMT to promote cancer invasiveness.

Furthermore, it was identified that the prognostic value of IMP3 expression in tumor cells was better than that of lymph node metastasis. Therefore, IMP3 may be a novel prognostic marker in CRC. The modern evolution of CRC treatment has tended toward multidisciplinary management combining multiple types of treatment, including chemotherapy, surgery and radiotherapy (30). Advances in preoperative chemotherapy and radiation have reduced disease recurrence and increased survival in high-risk diseases (31). However, preoperative chemotherapy and radiotherapy can lead to partial or complete pathological regression (10-27\%) of CRC (32). The present data suggested that IMP3 expression in in biopsy specimens form patients with CRC may be used as a parameter to select patients that are most likely to benefit from preoperative chemotherapy and radiotherapy.

Finally, the present results suggested that CRCs may benefit from a targeted anti-IMP3 therapy on the basis of the finding that IMP3 was expressed mainly in tumor cells (72.3\%). Since benefit was seen in survival in metastatic CRC with the therapeutic strategy of targeting tumor stroma (2), the finding from the present study that stromal expression of IMP3 is associated with advanced tumor TNM stage and metastasis may suggest that stromal therapy may be a viable approach for CRC. We hypothesize that targeting a tumor as an organ (i.e. the tumor and stroma together) would be more effective than targeting the tumor cells alone.

Here, the findings suggested that IMP3 expression was upregulated in the tumor cells and stromal compartments of patients with CRC compared with that in the normal colonic mucosal epithelium and the colonic adenoma. A significant association was identified between stromal IMP3 expression and lymph node metastasis and advanced tumor TNM stage. Moreover, IMP3 overexpression in tumor cells can be used as an independent factor for predicting poor prognosis of patients with CRC. However, although the results presented here may be useful as a biological marker, the findings may not specifically reflect the biological nature of cancer is only a preliminary phenomenological study and does not investigate the specific molecular mechanism (33). Further research of the underlying interactive mechanism of IMP3 in primary tumor and stroma should allow an improved understanding of more specific interactions between tumor cells and microenvironment. A larger scale prospective study will be necessary to verify the prognostic significance of IMP3.

\section{References}

1. Torre LA, Bray F, Siegel RL, Ferlay J, Lortet-Tieulent J and Jemal A: Global cancer statistics, 2012. CA Cancer J Clin 65: 87-108, 2015.

2. Isella C, Terrasi A, Bellomo SE, Petti C, Galatola G, Muratore A, Mellano A, Senetta R, Cassenti A, Sonetto C, et al: Stromal contribution to the colorectal cancer transcriptome. Nat Genet 47 : 312-319, 2015.

3. Giordano G, Febbraro A, Venditti M, Campidoglio S, Olivieri N, Raieta K, Parcessepe P, Imbriani GC, Remo A and Pancione M: Targeting angiogenesis and tumor microenvironment in metastatic colorectal cancer: Role of aflibercept. Gastroenterol Res Pract 2014: 526178, 2014.

4. Müeller-Pillasch F, Lacher U, Wallrapp C, Micha A, Zimmerhackl F, Hameister H, Varga G, Friess H, Büchler M, Beger HG, et al: Cloning of a gene highly overexpressed in cancer coding for a novel $\mathrm{KH}$-domain containing protein. Oncogene 14: 2729-2733, 1997.

5. Liao B, Hu Y, Herrick D and Brewer G: The RNA-binding protein IMP-3 is a translational activator of insulin-like growth factor II leader-3 mRNA during prolifearation of human K562 leukemia cells. J Biol Chem 280: 18517-18524, 2005.

6. Bell JL, Wächter K, Mühleck B, Pazaitis N, Köhn M, Lederer M and Hüttelmaier S: Insulin-like growth factor 2 mRNA-binding proteins (IGF2BPs): Post-transcriptional drivers of cancer progression? Cell Mol Life Sci 70: 2657-2675, 2013.

7. Gong Y, Woda BA and Jiang Z: Oncofetal protein IMP3, a new cancer biomarker. Adv Anat Pathol 21: 191-200, 2014. 
8. Mueller F, Bommer M, Lacher U, Ruhland C, Stagge V, Adler G, Gress TM and Seufferlein T: KOC is a novel molecular indicator of malignancy. Br J Cancer 88: 699-701, 2003.

9. Yuan RH, Wang CC, Chou CC, Chang KJ, Lee PH and Jeng YM: Diffuse expression of RNA-binding protein IMP3 predicts high-stage lymph node metastasis and poor prognosis in colorectal adenocarcinoma. Ann Surg Oncol 16: 1711-1719, 2009.

10. Li D, Yan D, Tang H, Zhou C, Fan J, Li S, Wang X, Xia J, Huang F, Qiu G and Peng Z: IMP3 is a novel prognostic marker that correlates with colon cancer progression and pathogenesis Ann Surg Oncol 12: 3499-3506, 2009.

11. Lochhead P, Imamura Y, Morikawa T, Kuchiba A, Yamauchi M, Liao X, Qian ZR, Nishihara R, Wu K, Meyerhardt JA, et al: Insulin-like growth factor 2 messenger RNA binding protein 3 (IGF2BP3) is a marker of unfavourable prognosis in colorectal cancer. Eur J Cancer 48: 3405-3413, 2012.

12. Lin L, Zhang J, Wang Y, Ju W, Ma Y, Li L and Chen L: Insulin-like growth factor-II mRNA-binding protein 3 predicts a poor prognosis for colorectal adenocarcinoma. Oncol Lett 6: 740-744, 2013.

13. Herrera M, Islam AB, Herrera A, Martín P, García V, Silva J, Garcia JM, Salas C, Casal I, de Herreros AG, et al: Functional heterogeneity of cancer-associated fibroblasts from human colon tumors shows specific prognostic gene expression signature. Clin Cancer Res 19: 5914-5926, 2013.

14. Fidler IJ: The pathogenesis of cancer metastasis: The 'seed and soil' hypothesis revisited. Nat Rev Cancer 3: 453-458, 2003.

15. Bhowmick NA and Moses HL: Tumor-stroma interactions. Curr Opin Genet Dev 15: 97-101, 2005.

16. Zhang J and Liu J: Tumor stroma as targets for cancer therapy. Pharmacol Ther 137: 200-215, 2013.

17. Massoner P, Haag P, Seifarth C, Jurgeit A, Rogatsch H, Doppler W, Bartsch $\mathrm{G}$ and Klocker $\mathrm{H}$ : Insulin-like growth factor binding protein-3 (IGFBP-3) in the prostate and in prostate cancer: Local production, distribution and secretion pattern indicate a role in stromal-epithelial interaction. Prostate 68: 1165-1178, 2008.

18. Kalluri R and Weinberg RA: The basics of epithelial-mesenchymal transition. J Clin Invest 119: 1420-1428, 2009.

19. Prall F: Tumour budding in colorectal carcinoma. Histopathology 50: 151-162, 2007.

20. Hase K, Shatney CH, Mochizuki H, Johnson DL, Tamakuma S, Vierra M and Trollope M: Long-term results of curative resection of 'minimally invasive' colorectal cancer. Dis Colon Rectum 38 : 19-26, 1995.

21. Bosman FT, Carneiro F, Hruban RH and Theise ND: WHO classification of tumours of the digestive system, Fourth Edition. WHO Classif Tumours Digestive System 3, 2010.
22. O'Connell JB, Maggard MA and Ko CY: Colon cancer survival rates with the new American Joint Committee on Cancer sixth edition staging. J Natl Cancer Inst 96: 1420-1425, 2004.

23. Karamitopoulou E, Zlobec I, Kölzer V, Kondi-Pafiti A, Patsouris ES, Gennatas K and Lugli A: Proposal for a 10-high-power-fields scoring method for the assessment of tumor budding in colorectal cancer. Mod Pathol 26: 295-301, 2013.

24. Bissell MJ and Radisky D: Putting tumours in context. Nat Rev Cancer 1: 46-54, 2001.

25. Calorin L and Bianchini F: Environmental control of invasiveness and metastatic dissenmination of tumor cells: The role of tumor cell-host cell interations. Cell Commun Signal 8: 24, 2010.

26. Sampson N, Zenzmaier C, Heitz M, Hermann M, Plas E, Schäfer G, Klocker H and Berger P: Stromal insulin-like growth factor binding protein 3 (IGFBP3) is elevated in the diseased human prostate and promotes ex vivo fibroblast-to-myofibroblast differentiation. Endocrinology 154: 2586-2599, 2013.

27. Tsujino T, Seshimo I, Yamamoto H, Ngan CY, Ezumi K, Takemasa I, Ikeda M, Sekimoto M, Matsuura N and Monden M Stromal myofibroblasts predict disease recurrence for colorectal cancer. Clin Cancer Res 13: 2082-2090, 2007.

28. Zlobec I and Lugli A: Epithelial mesenchymal transition and tumor budding in aggressive colorectal cancer: Tumor budding as oncotarget. Oncotarget 1: 651-661, 2010

29. Giger OT, Comtesse SC, Lugli A, Zlobec I and Kurrer MO Intra-tumoral budding in preoperative biopsy specimens predicts lymph node and distant metastasis in patients with colorectal cancer. Mod Pathol 25: 1048-1053, 2012.

30. van de Velde CJ, Aristei C, Boelens PG, Beets-Tan RG, Blomqvist L, Borras JM, van den Broek CB, Brown G, Coebergh JW, Cutsem EV, et al: EURECCA colorectal: Multidisciplinary mission statement on better care for patients with colon and rectal cancer in Europe. Eur J Cancer 49: 2784-2790, 2013

31. Ahmed S, Johnson $\mathrm{K}$, Ahmed $\mathrm{O}$ and Iqbal $\mathrm{N}$ : Advances in the management of colorectal cancer: From biology to treatment. Int J Colorectal Dis 29: 1031-1042, 2014.

32. Maas M, Nelemans PJ, Valentini V, Das P, Rödel C, Kuo LJ, Calvo FA, García-Aguilar J, Glynne-Jones R, Haustermans $\mathrm{K}$, et al: Long-term outcome in patients with a pathological complete response after chemoradiation for rectal cancer: A pooled analysis of individual patient data. Lancet Oncol 11: 835-844, 2010.

33. Nishio K, Inoue A, Qiao S, Kondo H and Mimura A: Senescence and cytoskeleton: Overproduction of vimentin induces senescent-like morphology in human fibroblast. Histochem Cell Biol 116: 321-327, 2001. 